\title{
Biodiversity mediates top-down control in eelgrass ecosystems: a global comparative-experimental approach
}

\author{
J. Emmett Duffy, ${ }^{1,2 *}$ Pamela L. \\ Reynolds, ${ }^{1}$ Christoffer Boström, ${ }^{3}$ \\ James A. Coyer, ${ }^{4}$ Mathieu Cusson, ${ }^{5}$ \\ Serena Donadi, ${ }^{6}$ James G. \\ Douglass, ${ }^{7}$ Johan S. Eklöf, ${ }^{8}$ \\ Aschwin H. Engelen, ${ }^{9}$ Britas \\ Klemens Eriksson, ${ }^{6}$ Stein \\ Fredriksen, ${ }^{10}$ Lars Gamfeldt, ${ }^{11}$ \\ Camilla Gustafsson, ${ }^{12}$ Galice \\ Hoarau, ${ }^{13}$ Masakazu Hori, ${ }^{14}$ Kevin \\ Hovel, ${ }^{15}$ Katrin Iken, ${ }^{16}$ Jonathan S. \\ Lefcheck, ${ }^{1}$ Per-Olav Moksnes, ${ }^{11}$ \\ Masahiro Nakaoka, ${ }^{17}$ Mary I. \\ O'Connor, ${ }^{18}$ Jeanine L. Olsen, ${ }^{6}$ \\ J. Paul Richardson, ${ }^{1}$ Jennifer L. \\ Ruesink, ${ }^{19}$ Erik E. Sotka, ${ }^{20}$ Jonas \\ Thormar, ${ }^{10}$ Matthew A. Whalen ${ }^{21}$ \\ and John J. Stachowicz ${ }^{21}$
}

\begin{abstract}
Nutrient pollution and reduced grazing each can stimulate algal blooms as shown by numerous experiments. But because experiments rarely incorporate natural variation in environmental factors and biodiversity, conditions determining the relative strength of bottom-up and top-down forcing remain unresolved. We factorially added nutrients and reduced grazing at 15 sites across the range of the marine foundation species eelgrass (Zostera marina) to quantify how top-down and bottom-up control interact with natural gradients in biodiversity and environmental forcing. Experiments confirmed modest top-down control of algae, whereas fertilisation had no general effect. Unexpectedly, grazer and algal biomass were better predicted by cross-site variation in grazer and eelgrass diversity than by global environmental gradients. Moreover, these large-scale patterns corresponded strikingly with prior small-scale experiments. Our results link global and local evidence that biodiversity and top-down control strongly influence functioning of threatened seagrass ecosystems, and suggest that biodiversity is comparably important to global change stressors.
\end{abstract}

\section{Keywords}

Biodiversity-ecosystem functioning, bottom-up control, coordinated experiments, food webs, metabolic ecology, structural equation modelling, top-down control.

Ecology Letters (2015) 18: 696-705

\section{INTRODUCTION}

Nutrient pollution and alteration of food webs by exploitation and invasion are two of the dominant human impacts on natural ecosystems. A wealth of evidence from controlled experiments confirms that each can be important in certain situations, fuelling a long-running debate about the conditions favouring bottom-up vs. top-down forcing and their management implications (Hunter \& Price 1992; Cloern 2001; Heck \& Valentine 2007; Gruner et al. 2008; Eriksson et al. 2009). Manipulative approaches have greatly advanced our understanding of how these processes shape experimental communities, but their implications for complex natural systems are often unclear. By design such experiments hold con-

${ }^{1}$ Virginia Institute of Marine Science, Gloucester Point, VA 23062-1346, USA

${ }^{2}$ Tennenbaum Marine Observatories Network, Smithsonian Institution,

Washington, D.C. 20013-7012, USA

${ }^{3}$ Department of Biosciences, Environmental and Marine Biology, Åbo Akademi University, 20520 Åbo, Finland

${ }^{4}$ Shoals Marine Laboratory, Cornell University, Portsmouth, NH 03801, USA

${ }^{5}$ Département des sciences fondamentales \& Québec-Océan, Université du Québec à Chicoutimi, Chicoutimi, QC G7H 2B1, Canada

${ }^{6}$ Centre for Ecological and Evolutionary Studies, University of Groningen, 9747 AG, Groningen, The Netherlands

${ }^{7}$ Florida Gulf Coast University, Fort Myers, FL 33965, USA

${ }^{8}$ Department of Ecology, Environment and Plant Sciences, Stockholm University, 10691 Stockholm, Sweden

${ }^{9}$ Centro de Ciências do Mar do Algarve (CCMAR), University of Algarve, 8005139 Faro, Portugal

${ }^{10}$ Department of Biosciences, University of Oslo, 0316 Oslo, Norway

${ }^{11}$ Department of Biological and Environmental Sciences, University of Gothen-

burg, SE-405 30 Göteborg, Sweden stant many other environmental and human factors known to strongly influence ecosystem processes, raising the question of how important the manipulated factors are relative to other drivers. These include, in particular, the spatial variation in environment and biodiversity that are characteristic of wild ecosystems but that are rigorously controlled in most experiments. Resolving these issues poses formidable challenges, requiring new approaches that can integrate the power of experiments with observational data on those regional processes that cannot be manipulated (Grace 2006; Cardinale et al. 2012). One such hybrid approach involves a coordinated experimental network, in which simple controlled experiments are replicated across space, allowing for the incorporation of environmental context into the analysis. Such networks have

${ }^{12}$ Tvärminne Zoological Station, University of Helsinki, 10900 Hanko, Finland

${ }^{13}$ Faculty of Biosciences and Aquaculture, University of Nordland, 8049 Bodø, Norway

${ }^{14}$ Fisheries Research Agency, 739-0452 Hiroshima, Japan

${ }^{15}$ Department of Biology, San Diego State University, San Diego, CA 92182, USA

${ }^{16}$ School of Fisheries and Ocean Sciences, University of Alaska Fairbanks, AK 99775, USA

${ }^{17}$ Akkeshi Marine Station, Field Science Center for Northern Biosphere, Hokkaido University, Aikappu, Akkeshi, Hokkaido 088-1113, Japan

${ }^{18}$ Department of Zoology and Biodiversity Research Centre, University of British Columbia, Vancouver, BC V6T 1Z4, Canada

${ }^{19}$ Department of Biology, University of Washington, Seattle, WA 98195, USA

${ }^{20}$ Grice Marine Laboratory, College of Charleston, Charleston, SC 29412, USA

${ }^{21}$ Department of Evolution and Ecology, University of California, Davis, CA, 95616, USA

*Correspondence: E-mail: duffye@si.edu 
made important advances, e.g. in understanding nutrient dynamics in terrestrial grasslands (Borer et al. 2014).

The Zostera Experimental Network (ZEN, www.zenscience.org) seeks to understand how complex regional and local processes interact to shape community and ecosystem structure by focusing on communities associated with the marine foundation species eelgrass (Zostera marina), which is distributed across a broad range of conditions and biogeographic provinces (Moore \& Short 2006). Eelgrass creates habitat for productive and economically important communities along coasts and estuaries throughout the northern hemisphere, supporting assemblages of epifaunal herbivores and detritivores, including small crustaceans, gastropod mollusks, and polychaete worms. These mesograzers feed on micro- and macroalgae that grow on seagrass blades and are important conduits of production to higher trophic levels including commercial and recreational fisheries (Valentine \& Duffy 2006). Seagrass dominance requires low biomass of competing algae, which is maintained by a combination of grazing and low nutrient conditions. This balance can be upset by food web perturbations that reduce grazing on algae, or by nutrient loading that stimulates algal accumulation, but the relative importance of these bottom-up and top-down factors in the dynamics of seagrass systems remains a subject of debate (Hughes et al. 2004; Heck \& Valentine 2007).

Complementing the long interest in relative importance of top-down and bottom-up forcing, a parallel line of research has focused on biodiversity - including species and genetic richness - as an important driver of ecosystem structure and functioning. Numerous experiments have now converged on the conclusion that declining variety of species, genetic lineages, and functional types generally results in declining ecosystem productivity and stability across a range of systems (Cardinale et al. 2011, 2012). In eelgrass systems specifically, prior experiments implicate both genetic and species richness in mediating the key interaction between grazers and epiphytic algae in eelgrass beds: plots planted with genotypically diverse eelgrass recruited denser fauna (Hughes \& Stachowicz 2004; Reusch et al. 2005), and plots seeded with species-rich grazer assemblages more effectively controlled epiphytic algae (Duffy et al. 2003, 2013). Yet it remains unclear how results from controlled experiments in mesocosms or single field sites translate to nature where many factors interact to drive productivity, trophic transfer, and other ecosystem processes (Srivastava \& Vellend 2005).

Community functional composition and richness are potentially central in mediating the strength of top-down control and responses to changing resources (Strong 1992; Polis \& Strong 1996; Oksanen \& Oksanen 2000; Duffy et al. 2007). We sought to link these research traditions by testing whether the signature of biodiversity effects is apparent across larger scales, in naturally variable ecosystems, and specifically whether they influence the relative importance of top-down vs. bottom-up forcing. To do so we conducted coordinated manipulations of nutrients and grazing across 15 sites spanning the northern hemisphere (Fig. 1a, Table S1) and encompassing the global spectrum of environments supporting eelgrass, including a natural gradient in grazer species richness from 5 to 27 species (Fig. S1). Our experimental treatments simulated two major human influences on coastal ecosystems: nutrient pollution and fishing-induced food web changes that weaken top-down control (Heck \& Valentine 2007; Duffy et al. 2013). Both types of perturbation have been demonstrated at various locations to cause blooms of epiphytic algae that compete with eelgrass, reducing its dominance (Duffy et al. 2013) and potentially tipping the system between states dominated by seagrass vs. epiphytic algae (Reynolds et al. 2014). We evaluated how these bottom-up and top-down processes are influenced by environment and biodiversity, specifically eelgrass genetic and grazer species diversity, of the local community using path analysis (Grace 2006), by integrating experimental data with natural variation among communities and controlling statistically for cross-site variation in biodiversity, environmental, and potential anthropogenic drivers.

We used path analysis to test the following hypotheses (Fig. 2a). First we expected that grazing impacts on plants should increase with environmental temperature (O'Connor 2009), and with plant nitrogen content (Mattson 1980). Based on prior experimental work we hypothesised that biodiversity (grazer species richness) should increase resistance to perturbations, in this case experimental nutrient addition, and that increasing grazer richness should increase grazer biomass and grazing pressure on algae (Duffy et al. 2003). Human influence is likely to have strong impacts on structure and functioning of eelgrass systems through a variety of mechanisms but the specific expectations for grazer and algal biomass depend on food web structure and the specific types of impact. Our comparative-experimental study demonstrated that grazer reduction has generally stronger effects on algal biomass in eelgrass ecosystems than does local nutrient addition, and that grazer and algal biomass were better predicted by cross-site variation in biodiversity than by global environmental gradients.

\section{MATERIALS AND METHODS}

\section{The ZEN field experimental module}

In summer 2011 we conducted identical field experiments at 15 sites (Fig. 1a, Table S1) encompassing the geographic range of eelgrass $\left(32-67^{\circ} \mathrm{N}\right)$ and a broad range in environmental conditions (Fig. S1). Each experiment was a two-way factorial experiment crossing nutrient fertilisation with crustacean mesograzer reduction. Forty plots were established at each site at depths between 0.5 and $3 \mathrm{~m}$ below mean low water. Each plot was defined by three PVC stakes forming a triangle $\sim 50 \mathrm{~cm}$ on a side. Crustacean grazer reduction was accomplished by attaching a plaster block containing the degradable insecticide carbaryl (Poore et al. 2009; Whalen et al. 2013), hereafter deterrent, or alternatively a plaster control block to each stake. Fertilisation was accomplished by attaching a mesh bag containing $300 \mathrm{~g}$ of slow-release fertiliser $(\mathrm{N}: \mathrm{P}: \mathrm{K}=14: 14: 14$, Plantacote, SQM VITAS, Amsterdam, Netherlands ${ }^{\mathrm{TM}}$ ), or an empty mesh control bag, to one pole of each plot. Thus, there were ten plots of each of the four combinations of fertiliser (present, absent) and grazer deterrent (present, absent) at each site. Carbaryl effects on 

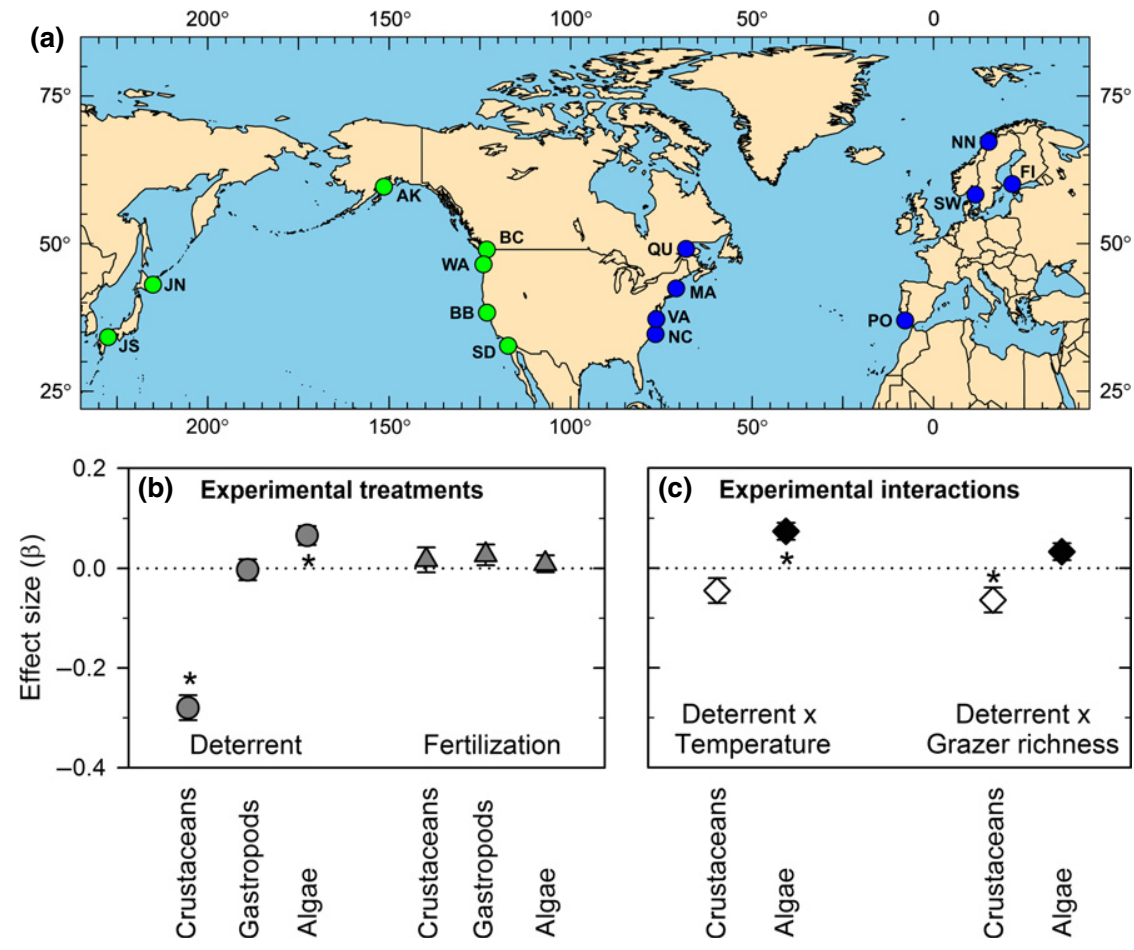

Figure 1 Effects of experimental fertilisation, grazer reduction, and their interactions with environment and biodiversity across the range of eelgrass. (a) Map of the 15 Zostera Experimental Network sites, with blue and green site codes (Table S1) identifying Atlantic (plus Baltic) and Pacific sites, respectively. (b) Mean ( \pm SEM) effects of grazer deterrent and fertilisation on log biomasses of crustacean and gastropod grazers and epiphytic microalgae, estimated as partial regression coefficients from the full path model (1). (c) Influence of cross-site variation in temperature and grazer richness on grazing impact (Deterrent effect), estimated from interaction terms in SEM models 11 and 13 (Tables 1, S2b, Fig. S3).

crustaceans extended $\sim 60 \mathrm{~cm}$ from the source (Whalen et al. 2013). Plots were separated by $>2 \mathrm{~m}$. The experiment was maintained for approximately 4 weeks at each site, a period long enough to see effects in pilot studies (Whalen et al. 2013).

At the end of the experiment we measured the abundance and species composition of epifauna, including mesograzers, in each plot by enclosing eelgrass and associated fauna in a $500 \mu \mathrm{m}$ mesh bag underwater, removing the fauna from the plants in the laboratory, and preserving in $70 \%$ ethanol. Epifaunal animals (excluding meiofauna) were identified and size-fractionated to estimate biomass using empirical equations (Edgar 1990). Faunal biomass was standardised to above-ground plant biomass (mg fauna $\mathrm{g}_{\mathrm{plant}}{ }^{-1}$ ). A separate eelgrass shoot was removed from each plot and scraped to obtain microalgal biomass ( $\mu \mathrm{g}$ chl $a \mathrm{~g}$ dry Zostera ${ }^{-1}$ ), which was measured spectrophotometrically or fluorometrically (Duffy et al. 2003). Biomasses of epiphytic algae, grazing crustaceans and grazing gastropods constituted the response variables of primary interest. Eelgrass leaf nitrogen content was quantified in standardised young leaf material from five pooled shoots in each plot at the end of the experiment using a CHN analyser (Thermo Fisher Scientific Inc., Waltham, MA).

\section{Site characteristics}

We collected data on several variables to assess how experimental effects varied across environmental context. At the site level, we measured water temperature throughout the experiment using HOBO Pendant ${ }^{\circledR}$ (Onset, Bourne, MA USA) temperature loggers, and salinity via refractometers. Eelgrass leaf $\% \mathrm{~N}$ was measured from ambient (control) plots and averaged across plots to obtain a proxy for sitelevel nutrient status (Burkholder et al. 2007). As a rough measure of anthropogenic influences, we obtained estimates of human population density near each site using the LandScan $^{\text {TM }}$ Global Population Database (Oak Ridge National Laboratory, Oak Ridge, TN USA), implemented in National Geographic's Mapmaker Interactive (http://mapmaker.education.nationalgeographic.com); this tool presents human population density on a 5-point ordinal scale (bins) from $<1$ to $>500$ per cell.

We measured two aspects of biodiversity shown previously to influence eelgrass ecosystem properties in experiments. Eelgrass genotypic richness at a site was calculated as the sum of unique genotypes found among the 40 plots. Mesograzer species richness was estimated at the site level as the sum of all species recorded across the set of 40 plots. We chose to aggregate diversity at the site level as we consider the list of species sampled at the site a better measure of the species potentially visiting a plot during the experiment than the single, final sampling point from that plot. To assess whether our richness estimates were affected by the number of individuals sampled, we employed fixed-coverage subsampling, a variant of rarefaction (Chao \& Jost 2012). This approach extrapolates site-level estimates of richness based on sample 'completeness,' or the proportion of individuals in the community estimated to belong to species detected by sampling, and thus can be used 

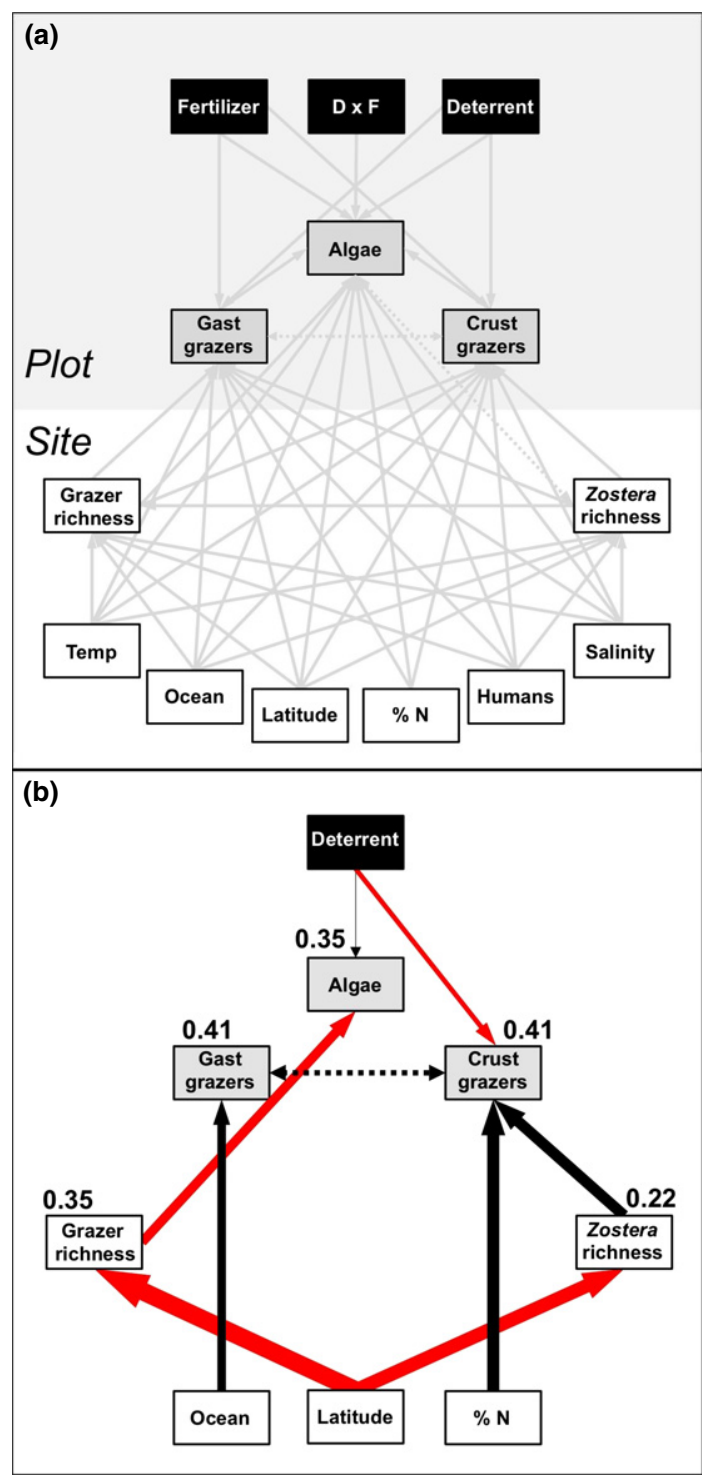

Figure 2 Path analysis of combined experimental and regional controls on grazer and algal biomass across the range of eelgrass. (a) Schematic representation of variables included in the model: those measured at site and plot levels are shown in lower white and upper grey sections of panel, respectively. Experimental treatments are black. Biomasses of crustacean (Crust) and gastropod (Gast) grazers and algae were modelled as a function of temperature (Temp), salinity, site nutrient status $(\% \mathrm{~N})$, latitude, ocean (Atlantic vs. Pacific), and richness of grazer species and eelgrass genotypes. (b) Best model 10 (AIC weight $=0.61$, Table 1). Thickness of black (positive) and red (negative) paths is proportional to range-standardised path coefficient. Marginal $R^{2}$ values are shown for endogenous variables. The double-headed, dashed arrow represents a correlated error rather than a hypothesised directed causal path.

to determine whether our efforts yielded richness estimates close to their asymptotic maximum.

All statistical analyses were conducted using R 3.03 (R Development Core Team 2014).

\section{Eelgrass genetic analyses}

Collection of eelgrass samples (40 ramets per site, i.e. one per plot), DNA extraction, microsatellite amplification and geno- typing followed Olsen et al. (2013). Six microsatellite loci (CT2, CT35, CT12, CT17D, CT19, CT20) were scored (Reusch et al. 1999; Reusch 2000). The numbers of genets sampled at a site was distinguished with GENClone 2.0 (Arnaud-Haond \& Khalid 2007), considering as identical only those genets with non-significant probabilities of identity by chance $\left[P \operatorname{sex}\left(F_{\mathrm{IS}}\right)\right]$. Genotypic richness, $R$ (number of unique genets, $G-1$ divided by the number of sampled ramets, $N-1$ ) was also calculated with GenClone 2.0. Here, we report values of genotypic richness per site (R).

\section{Path analysis of combined experimental and observational data}

We expressed hypotheses about the integrated functioning of the eelgrass ecosystem as graphical networks of interaction paths, and analysed each as a set of linked equations using path analysis (a variant of the broader field of structural equation models, but which uses only observed rather than latent variables, Fig. 2a, and Fig. S3). An advantage of SEM for studying complex systems is that, by linking together component models for different response variables, it allows rigorous estimation of indirect effects and tests of the overall fit of a complex, causal network of influence (Grace 2006). Models were fit using data from the 40 plots at each of 15 sites for a total of 600 sampling units. Predictors included the following exogenous variables, i.e. whose variance arose outside the model: (1) experimental treatments (grazer deterrent, fertilisation, their interaction and unmanipulated control), (2) biogeographic variation [latitude, ocean (Atlantic vs. Pacific)], (3) abiotic forcing factors, including temperature, salinity, nutrient availability proxied as eelgrass \% $\mathrm{N}$ (Burkholder et al. 2007), and (4) human population density. Endogenous variables are those whose variation the model seeks to explain (response variables), including in our case biodiversity (eelgrass genotypic richness, grazer species richness), and biomasses of crustacean grazers, gastropod grazers, and algae.

Preliminary exploration found that simple models with few explanatory variables had poor overall fit via $D$-separation tests (see below). Therefore, we developed a set of candidate models by first removing paths from the 'full' model (containing nearly all possible paths, Fig. 2a, Table 1) to test their hypothesised importance. Next, we used the best fitting models from this process and added interaction terms to test specific hypotheses about how bottom-up and top-down processes should vary with latitude, temperature and biodiversity. Including the full model 1 incorporating all paths of interest, we fit 14 candidate models that tested the influence of the experimental and environmental variables listed above on biomasses of algae, crustacean and gastropod grazers (models 210 ), along with interactions motivated by theory (models 1114, Table 1, Fig. S3). To test the metabolic hypothesis that grazing impacts (measured as effect of grazer deterrent on algae) should increase with environmental temperature, model 11 incorporated an interaction between grazer deterrent and temperature. We also tested the related hypothesis that grazer impacts should decrease with latitude by incorporating an interaction of deterrent effect and latitude (model 12). We tested the hypothesis that grazing is more effective where grazer richness is higher by incorporating an interactive effect of 
grazer richness and grazer deterrent on algae (model 13); the hypothesis is supported if the interaction is significant and positive. Finally, model 14 tested the hypothesis that grazer richness promotes resistance against nutrient loading; this hypothesis is supported if fertilisation increases algal biomass less at sites with higher grazer richness (negative interaction of grazer richness and fertilisation effect on algae). Because we used mean leaf $\%$ nitrogen of control plots as a proxy for site nutrient status in the path analyses, effects of experimental treatments on leaf $\%$ nitrogen were estimated separately across sites.

Our study design produced hierarchical (i.e. multilevel) data at two nested levels (40 plots within each of 15 sites, for a total of 600 plots). As a result the analysis proceeded in two phases. In the first phase, we sought to explain variation in the two endogenous variables measured at the site level, i.e. grazer species richness and eelgrass genotypic richness (all other endogenous variables were measured at the plot level). Because these two biodiversity variables were measured at the site level (taking 15, rather than 600, unique values), they had no hierarchical structure and were therefore modelled using standard multiple linear regressions. This analysis showed that the only significant predictor of grazer and eelgrass richness was latitude (compare models 1 and 2, Table 1), so the influence of latitude was retained as the sole predictor of grazer species richness and eelgrass genotypic richness in all subsequent models (models 3-14) that focused on the plot-level variables of algal and grazer biomasses.

In the second, main phase of analysis we sought to understand the drivers of plot-level biomasses of algae and crustacean and gastropod grazers, corresponding to the hypotheses described in Table 1. Because some predictor variables were measured at the plot level (with 600 unique values) and others at the site level (with 15 unique values), we modelled the responses with linear mixed effects models, including a random effect modelling variation in intercepts among the 15 sites. In this phase we used Shipley's (2009) approach to estimating a multilevel path model using directional separation (D-sep) tests. This approach constructs the path model as a set of hierarchical linear mixed models, each of which was fit using restricted maximum likelihood with the nlme package (version 3.1-117) in R, and the overall path model (the SEM) was fit using the R package piecewiseSEM (Lefcheck \& Duffy 2015). Prior to fitting the models we graphically examined distributions of all variables for outliers and severe departures from normality. Most biological variables were $\log _{10}$-transformed to improve normality. One path in the final model, connecting biomasses of crustaceans and gastropods (Fig. 2b), was considered a correlated error rather than a directed causal path because the positive coefficient seemed best interpreted as reflecting parallel responses to unmeasured forcing variables.

We selected among candidate models using two criteria. First, goodness of fit was estimated for each path model using Shipley's (2009) test of directional separation (D-sep), which combines the significance of unrealised paths into a single, Chi-squared distributed Fisher's $C$ statistic. For each candidate model that passed this test of adequate fit $(P>0.05)$ we then computed an Akaike Information Criterion (AIC) value using Shipley's (2013) general approach to computing AIC in path analyses. Finally, AIC weights were calculated and compared to evaluate the relative support for each candidate model. We graphically assessed the validity of model assumptions by plotting the residuals against the fitted values of each component model.

Once a model was chosen, we compared the relative importance of its predictor variables using standardised path coefficients. We standardised coefficients to the relevant ranges of the component variables as recommended by Grace (2006). A raw coefficient $\beta_{x y}$ expressing the effect of $\mathrm{x}$ on $\mathrm{y}$ is rangestandardised as $\beta$ range $_{x y}=\beta_{x y} \times\left(\mathrm{x}_{\max }-\mathrm{x}_{\min }\right) /\left(\mathrm{y}_{\max }-\mathrm{y}_{\min }\right)$, where the max and min values represent the largest and smallest values of the variables recorded in the data set (see Fig. S1). This approach produces a dimensionless coefficient that is easily interpretable in the original units. For example a $\beta$ value of -0.349 for effect of grazer richness on microalgal biomass means that microalgal biomass is expected to decline by $35 \%$ of its measured range as one moves across the entire

Table 1 Candidate path models (see Fig. S3 for structures)

\begin{tabular}{|c|c|c|c|c|c|c|}
\hline Model & Explanation & Fit $(P)$ & $\mathrm{K}$ & $\mathrm{AIC}$ & Delta AIC & AIC Wt \\
\hline 1 & Full model & 0.83 & 60 & 122.8 & 47.3 & 0.00 \\
\hline 2 & Latitude model: grazer and eelgrass richness affected only by latitude & 1.00 & 50 & 106.4 & 30.8 & 0.00 \\
\hline 3 & Latitude model minus experimental fertilisation effects & 0.96 & 44 & 101.4 & 25.8 & 0.00 \\
\hline 4 & Latitude model minus experimental grazer deterrent effects & 0.00 & 44 & 233.2 & 157.6 & 0.00 \\
\hline 5 & Latitude model minus biodiversity effects on grazer or algal biomass & 0.82 & 44 & 110.8 & 35.2 & 0.00 \\
\hline 6 & Latitude model minus salinity effects & 1.00 & 47 & 102.8 & 27.2 & 0.00 \\
\hline 7 & Latitude model minus human population density effects & 0.97 & 47 & 99.7 & 24.1 & 0.00 \\
\hline 8 & Latitude model minus fertiliser, salinity, human density effects & 0.98 & 38 & 82.5 & 6.9 & 0.02 \\
\hline 9 & Model 8 minus latitude effect on grazer or algal biomass & 0.98 & 35 & 77.6 & 2.0 & 0.22 \\
\hline 10 & Pruned model: Model 9 minus deterrent effect on gastropod biomass & 0.98 & 34 & 75.6 & 0.0 & 0.61 \\
\hline 11 & Pruned model plus interaction: deterrent $\times$ temperature & 0.99 & 36 & 79.8 & 4.2 & 0.07 \\
\hline 12 & Pruned model plus interaction: deterrent $\times$ latitude & 0.98 & 38 & 93.7 & 18.1 & 0.00 \\
\hline 13 & Pruned model plus interaction: deterrent $\times$ grazer richness & 0.99 & 36 & 80.0 & 4.4 & 0.07 \\
\hline 14 & Pruned model plus interaction: fertilisation $\times$ grazer richness & 0.94 & 37 & 83.9 & 8.3 & 0.01 \\
\hline
\end{tabular}

Fit was determined using D-separation tests. Models were compared via the Akaike Information Criterion (AIC), estimated from D-separation tests (Shipley 2013). Delta AIC is the difference in AIC score relative to the model with the lowest value (most parsimonious model) and AIC Weight (Wt) is the relative support for the model. Models in bold had AIC Weight $>0.05$ (see Materials and Methods for details). 
measured range of grazer richness, when the influence of other variables is controlled for. Marginal $R^{2}$ values for endogenous variables were calculated from the best model (model 10) using an approach designed for hierarchical mixed models (Nakagawa \& Schielzeth 2013).

Finally, to visualise key relationships among variables in our best model while accounting for the influence of other covariates, we generated partial correlation plots. The partial correlation between $\mathrm{X}$ and $\mathrm{Y}$ measures the association between $\mathrm{X}$ and $\mathrm{Y}$ when effects of all other variables in the model have been controlled statistically; it is visualised by plotting $r_{(X \mid \text { others })}$ vs. $r_{(Y \mid \text { others })}$, where $r_{(X \mid \text { others }}$ consists of the residuals of $\mathrm{X}$ from the linear model that regresses $\mathrm{X}$ on all variables included in the final model except for Y. Similarly, $r_{(Y \mid o t h e r s)}$ are the residuals of the model that regresses $Y$ on all variables except $X$. Where the model contains only sitelevel predictors the residuals used to calculate the partial correlation will take only as many values as there are sites (15 in our case) on the $\mathrm{x}$ axis (Fig. 3a, c), whereas when the model contains a predictor measured at the plot level (e.g. crustacean biomass) the residuals are not so constrained (Fig. 3b, d).

\section{Meta-analysis of prior experiments}

To explore parallels between the cross-site patterns found in our study and those of previous small-scale experiments, we extracted and summarised data from previous experiments manipulating biodiversity and/or nutrient fertilisation in eelgrass systems (Table S3). We focused on experiments that manipulated eelgrass genotypic richness, species richness of eelgrass-associated mesograzers, and/or experiments that measured responses of both algae and grazers to nutrient fertilisation (references are cited in Table S3). For comparison on a common scale, all values were standardised to a range

(a)

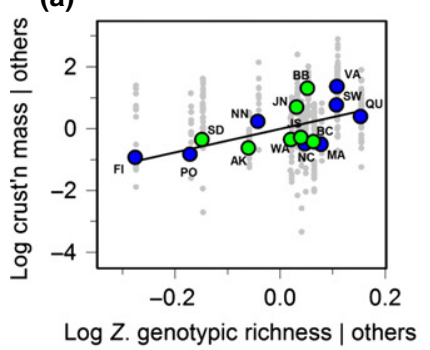

(e)

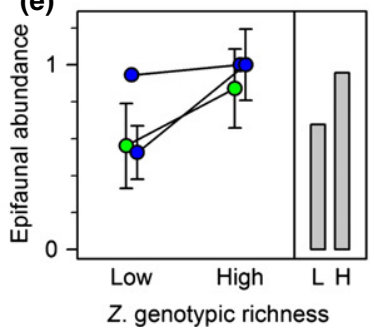

(b)

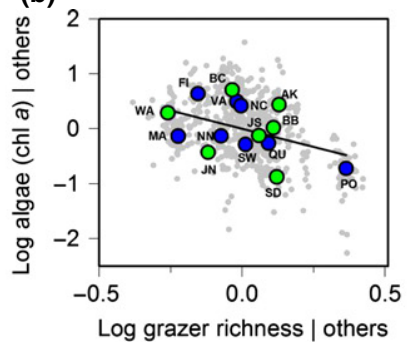

(f)

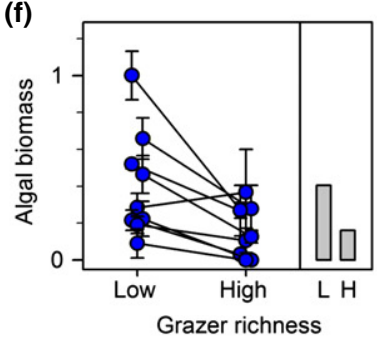

of zero to one by dividing each value by the highest value within that study.

\section{RESULTS}

The coordinated experiment revealed stronger top-down control of microalgae by grazers than bottom-up control by fertilisation, on average, across the geographic range of eelgrass (Fig. 1). This pattern of top-down control was also supported by path analysis showing negative covariation of algae and grazer assemblages across the 15 sites. Path analyses integrating the experimental (within-site) and observational (crosssite) data produced two very similar models which, compared with the other 12 models examined, had a combined AIC weight of 0.83 (Models 9 and 10, Table 1, Fig. 2b). Two additional models estimated to test the a priori hypotheses of interactions of grazer deterrent with temperature (model 11) and grazer richness (model 13) had poorer overall fit (each with AIC weight of 0.07 ) but significant interaction paths (Table S2b). All four models included a highly significant negative effect of deterrent on biomass of grazing crustaceans, and a concomitant positive, albeit weak effect on epiphytic microalgae (Fig. 1b, Table S2a, b). In contrast, none of the best models included a significant effect of experimental fertilisation on algal biomass (Fig. 1b, Table S2b), and removal of paths representing fertilisation effects improved model fit (i.e. AIC, compare model 3 with 2, Table 1). The importance of top-down control of algae by mesograzers is further emphasised by the model (4) that omitted the path between deterrent and grazer biomass, which had by far the least explanatory power of any candidate model (delta AIC $=157)$. The absence of experimental fertilisation effects on microalgae or grazers raises the question whether the treatment was adequate to raise local nutrient levels. A separate analysis suggests that it

(c)

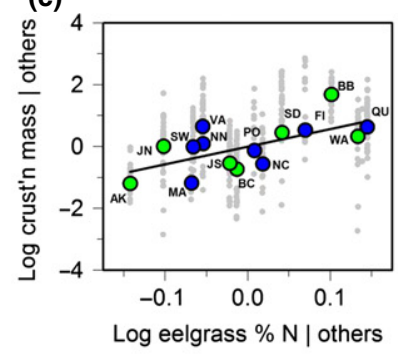

(g)

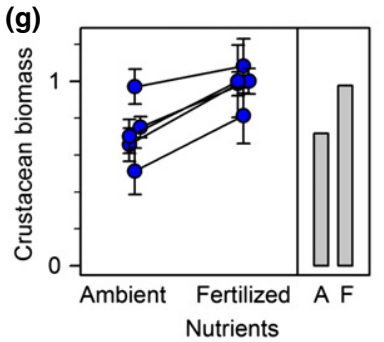

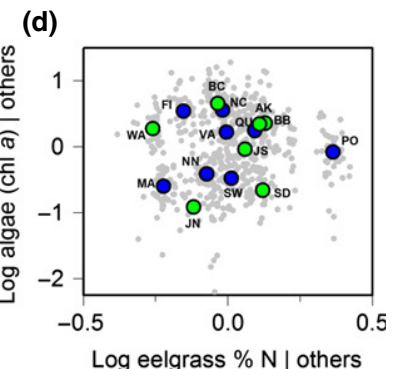

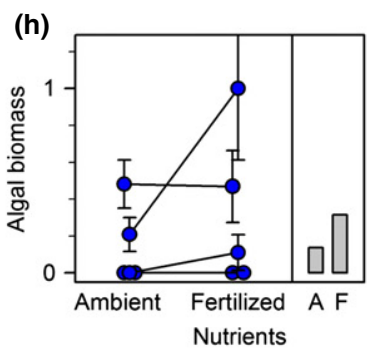

Figure 3 Predictors of algal and grazer biomass in global comparisons (this study) and prior plot- or mesocosm-scale experiments. (a-d) Partial correlations from the best SEM (model 10, Fig. 2b, Table S2), i.e. influence of a predictor when other variables ('others') are controlled statistically. Grey symbols denote values (model residuals) for individual plots, larger symbols are site means. (e-h) Results of prior experiments. Values are standardised (see Methods) and bars show mean values across the studies summarised in Table $\mathrm{S} 3$ ( $\mathrm{L}=$ low, $\mathrm{H}=$ high, $\mathrm{A}=$ ambient, $\mathrm{F}=\mathrm{fertilised}$ ). 
was, since experimental fertilisation did increase average eelgrass nitrogen content $(P=0.044$, Fig. S2).

The dominance of top-down vs. bottom-up control of algae in the experiments was mirrored by patterns in the observational data, i.e. cross-site relationships among nutrient status, grazers, and algal biomass. Specifically, none of the best path models showed a significant path from eelgrass nitrogen content (a proxy for site nutrient status) to algal biomass, whereas all included a strong effect of this proxy for nutrient status on crustacean grazer biomass (Fig. 2b, Table S2). The latter result confirms that, as in the experiment, variation in site nutrient status was ecologically significant but did not affect local algal biomass, presumably due to efficient trophic transfer of the nitrogen through algae to grazers as a result of strong top-down control by grazers.

We tested four a priori hypotheses to explain geographic variation in top-down control by fitting interactions between grazing impact (estimated as deterrent effect on algae) and environmental factors or biodiversity. Model 11, testing the role of temperature on grazing, revealed a strong positive interactive effect of temperature and deterrent on algae $(P<0.001)$, suggesting that top-down control generally strengthened in warmer areas (Fig. 1c). Model 13, testing how grazing varied with grazer richness, showed a tendency for higher grazing impact (i.e. more positive deterrent effect on algae) at sites with higher grazer richness $(P=0.051$, Fig. 1 c) . Although the paths corresponding to these a priori hypotheses were supported, the models had considerably lower AIC support (AIC weight $=0.07$ for each) compared with the best model 10. Model 14's hypothesis that more diverse grazer assemblages confer resistance to nutrient loading was not supported, as indicated by the poor fit of this model, which included a negative interaction between grazer richness and fertilisation effect on algae (Table 1, Fig. 1c). Model 12, testing whether grazing impact declined with latitude, independent of covarying gradients in temperature, was also poorly supported (Table 1, Fig. 1c), suggesting that temperature alone is a better predictor of grazing impact than latitude.

Analysis of cross-site patterns indicated that top-down impacts on algal biomass were mediated primarily by variation in grazer species richness rather than by variation in grazer biomass (Fig. 2b). Indeed the best-supported path models revealed, surprisingly, that eelgrass genotypic richness and grazer species richness were equally strong predictors of algal and grazer biomass as were among-site differences in resource (nitrogen) status. The biodiversity variables also were stronger predictors than variation across the global range in temperature or salinity, neither of which significantly affected grazer or algal biomass (Fig. 2b, Table S2). A model (5) that omitted effects of biodiversity (grazer species richness and eelgrass genotypic richness) on algal and grazer biomass had an AIC weight near zero (Table 1). Both grazer species richness and eelgrass genotypic richness declined with increasing latitude, and the models show that these large-scale gradients in biodiversity were the dominant direct influences on grazer and algal biomass (Fig. 2b). Sites with more genotypically diverse eelgrass supported higher biomass of crustacean grazers (Figs $2 \mathrm{~b}$ and $3 \mathrm{a}$ ) and sites with higher grazer species richness supported lower algal biomass (Figs $2 \mathrm{~b}$ and $3 \mathrm{~b}$ ); indeed natu- ral variation in grazer richness had an order of magnitude stronger effect on algae in the model than did variation in grazer biomass (Table S2). Finally, sites with more grazer species supported higher biomass of gastropod grazers (Fig. 2b).

When effects of biodiversity were isolated by controlling statistically for other factors using partial correlations, the strong influences of biodiversity emerging from cross-site comparison (100 to 1000 s of $\mathrm{km}$ ) in our analysis were strikingly similar to patterns documented in prior experiments comparing mesocosm or replicate field plots separated by metres to 10s of metres (Fig. 3a, b, Table S3). These cross-site comparisons also corroborate prior results showing that experimental fertilisation tends to raise biomass of crustacean grazers but not of algae (Fig. 3c, d).

\section{DISCUSSION}

Our coordinated experiment showed that removing grazers had a considerably stronger average effect on microalgal biomass than did local addition of nutrients at sites across the global range of eelgrass ecosystems, and that the importance of grazers in controlling algae increased with temperature and with species richness of the grazer assemblage. Integrated analysis of experimental and observational data via path analysis revealed two surprising results. First, the influence of cross-site variation in biodiversity (eelgrass genotypes and mesograzer species richness) and environmental drivers generally overwhelmed experimental effects of grazer and nutrient manipulation across the global range. Eelgrass and grazer richness were as strong predictors of algal and grazer biomass as differences in resource (nitrogen) status, and were stronger predictors than temperature or salinity, neither of which affected biomass in the best models. Path analysis showed that latitude explained variation in biodiversity, which in turn strongly influenced both plant and animal biomass. In contrast, the major environmental factor varying with latitude, temperature, had no main effect on algal and grazer biomass after controlling for grazer species richness (Fig. 2b, Table S2) although top-down control tended to be stronger at warmer sites (Fig. 1c).

Second, the strong influences of biodiversity in our global analysis were strikingly similar to results of prior small-scale experiments. This concordance addresses the common concern that biodiversity effects on functioning documented in experiments may derive from simplified conditions of questionable relevance to nature (Srivastava \& Vellend 2005). Several points illustrate the match between our observational data, comparing sites around the globe, and prior small-scale experimental data. First, the higher crustacean biomass at sites with more genotypically diverse eelgrass (Fig. 3a) matches previous experimental findings of increased invertebrate abundance in genotypically diverse eelgrass (Fig. 3e). This can be due to greater eelgrass biomass in genotypically diverse plots (Hughes \& Stachowicz 2004; Reusch et al. 2005), or because growth of individual herbivores is greater on diets of mixed genotypic composition (Tomas et al. 2011). Second, the lower algal biomass at sites with more grazer species (Fig. 3b) mirrors the stronger control of algal growth by diverse grazers in numerous eelgrass mesocosm experiments (Fig. 3f). Although 
we were unable to collect data on change in eelgrass biomass in our experiments, small-scale experiments find that stronger grazing on algae does increase eelgrass growth (Hughes et al. 2004; Reynolds et al. 2014).

This concordance of patterns from previous experiments and from our global comparative analysis also extended to bottomup control of grazer production. Nutrient status, proxied by eelgrass tissue nitrogen content (Burkholder et al. 2007), was strongly positively related to crustacean biomass but unrelated to algal biomass in comparisons across sites (Fig. 2b and 3c, d). Similarly, in prior experiments, fertilisation consistently increased the biomass of eelgrass-associated grazers, but not of epiphytic algae (Moksnes et al. 2008; Spivak et al. 2009; Baden et al. 2010) (Fig. 3g, h). This pattern of fertilisation 'bypassing' plants to increase grazers suggests that nitrogen enhances algal productivity, but that (initially at least) it is immediately grazed and channelled into increasing herbivore biomass. Why then did experimental fertilisation not increase crustacean biomass in our plots (Fig. 1b, c, Fig. 2b)? The most likely explanation is that these epifaunal crustaceans are sufficiently mobile that they dispersed from the small plots or were consumed by predators, resulting in no net accumulation of grazer biomass. In contrast, previous experiments used cages or mesocosms, which prevent the predation or export of nutrient-stimulated grazer production. Our results thus reinforce prior findings that moderate nutrient loading often fails to stimulate algal blooms when natural grazing pressure is maintained (Heck \& Valentine 2007; Eriksson et al. 2009; Hughes et al. 2013) and that intact food webs may provide resistance against eutrophication in seagrass ecosystems.

The effects of biodiversity in our global analyses derive from comparisons among sites differing naturally in eelgrass and grazer richness, raising the possibility that these effects might not be causal. We consider this unlikely for several reasons. First, our results corroborate theory predicting that higher grazer richness both depresses producer biomass and increases grazer biomass (Holt \& Loreau 2002). Second, the patterns we observed match those documented at smaller scales in multiple controlled experiments in eelgrass (Fig. 3, Table S3) and other systems (Cardinale et al. 2012). Third, the mechanisms involved should apply across scales: control of algae by diverse grazers involves both differences in feeding biology among species (complementarity) and dominance of strong grazer species (sampling effects) (Duffy et al. 2003; Best et al. 2013), both of which seem equally likely to operate at regional and small plot scales. Fourth, the effects of biodiversity in our study emerged from path models that controlled statistically for many potentially confounding influences including latitude, temperature and nutrients. Finally, a mechanistic link between grazer richness and top-down control is also consistent with the positive, albeit marginally significant, interactive effect of deterrent and grazer richness on algae (model 13, $P=0.051$ ), indicating that grazing pressure tends to be stronger - and grazer deterrent is thus more effective at stimulating algal accumulation - at sites with high grazer richness.

It is also conceivable that the direction of causality might be opposite that of our predictions in some cases. Specifically, environmental conditions promoting higher abundance might also accumulate more species (rather than vice versa as we have hypothesised) because a larger random sample of individuals tends to contain more species from a given source pool. To test for such artifacts we employed a fixed-coverage subsampling approach (Chao \& Jost 2012). This analysis revealed that observed richness ranged from 89 to $100 \%$ completeness across our 15 sites, with a mean of $98 \%$, strongly suggesting that our estimates of grazer richness are robust and comparable among sites. Moreover, such sampling effects could not easily explain the negative relationship, across trophic levels, of grazer richness with algal biomass (Fig. 3), i.e. it is unclear how low algal biomass could promote high grazer richness via sampling effects. Nor is high grazer biomass likely to enhance eelgrass genotypic richness since most herbivores in our system do not consume eelgrass. Instead our results seem best explained by higher richness promoting more effective resource use as predicted by theory (Holt \& Loreau 2002) and demonstrated in numerous experiments (Cardinale et al. 2012).

Our results have implications for coastal management, which has focused extensively on the threat posed by nutrient loading and associated algal blooms to seagrasses and other coastal habitats (Cloern 2001; Burkholder et al. 2007). Surprisingly, we found little evidence that local fertilisation increased epiphytic microalgae (Fig. 1b), and sites with higher nitrogen availability supported more grazing crustaceans but not more algae (Figs $2 \mathrm{~b}$ and $3 \mathrm{c}, \mathrm{d}$ ), despite wide variation in nutrient status (Fig. S1). Thus, while sustained nutrient loading can clearly have detrimental impacts on submerged vegetation (Cloern 2001; Burkholder et al. 2007), both our experimental results and our cross-site comparisons bolster the growing consensus that food web perturbations can have comparable or even greater impacts on estuarine producer biomass compared with moderate nutrient increases (Heck \& Valentine 2007; Eriksson et al. 2009; Baden et al. 2010; Hughes et al. 2013).

To inform management effectively in an era of global change, ecology must move beyond demonstrating which processes occur to quantifying their importance and interactions under ambient conditions. Our comparative-experimental analysis shows that biodiversity is a strong predictor of fundamental ecosystem processes, including producer and consumer biomass accumulation, in naturally complex field ecosystems on a global scale and is comparable in importance to largescale gradients in temperature, salinity and nutrients. Moreover, these processes appear related to biodiversity at both genetic and species levels. The qualitative concordance of our results, and other recent large-scale observations (Frank et al. 2007; Paquette \& Messier 2010; Mora et al. 2011; Maestre et al. 2012; Gamfeldt et al. 2013), with results of small-scale experiments supports the emerging conclusion that biodiversity is a fundamental controller of how ecosystems work, and is of comparable quantitative importance to major environmental drivers of metabolism, organismal fitness, and ecosystem processes (Cardinale et al. 2012; Hooper et al. 2012).

\section{ACKNOWLEDGEMENTS}

We thank the many staff, students, and volunteers who assisted with field and laboratory research; Jim Grace and 
Jarrett Byrnes for advice on structural equation modelling; Brad Cardinale and Jonathan Losos for comments that improved the MS; and the home institutions of all project partners, who provided invaluable in-kind support. Primary funding was provided by the National Science Foundation (OCE-1031061 to J.E.D.).

\section{AUTHORSHIP}

J.E.D. designed the study; J.E.D. and P.L.R. coordinated the Zostera Experimental Network; P.L.R. and J.P.R. led logistics; all authors performed field research and laboratory analyses; J.L.O. and J.A.C. conducted the genetic analyses; J.E.D., P.L.R., and J.S.L. analysed the data; J.E.D. drafted the manuscript, with substantial input from P.L.R., and J.J.S.; C.B., J.A.C., M.C., J.S.E., B.K.E., S.F., L.G., C.G., M.H., K.H., K.I., P.-O.M., M.N., J.S.L., M.I.O, P.L.R., J.P.R., J.L.R., E.E.S., J.J.S., J.T., M.A.W. commented on and edited the MS.

\section{REFERENCES}

Arnaud-Haond, S. \& Khalid, B. (2007). GENCLONE: a computer program to analyse genotypic data, test for clonality and describe spatial clonal organization. Mol. Ecol. Notes, 7, 15-17.

Baden, S., Boström, C., Tobiasson, S., Arponen, H. \& Moksnes, P.-O. (2010). Relative importance of trophic interactions and nutrient enrichment in seagrass ecosystems: a broad-scale field experiment in the Baltic-Skagerrak area. Limnol. Oceanogr., 55, 1435.

Best, R.J., Caulk, N.C. \& Stachowicz, J.J. (2013). Trait vs. phylogenetic diversity as predictors of competition and community composition in herbivorous marine amphipods. Ecol. Lett., 16, 72-80.

Borer, E.T., Seabloom, E.W., Gruner, D.S., Harpole, W.S., Hillebrand, H., Lind, E.M. et al. (2014). Herbivores and nutrients control grassland plant diversity via light limitation. Nature, 508, 517-520.

Burkholder, J., Tomasko, D. \& Touchette, B. (2007). Seagrasses and eutrophication. J. Exp. Mar. Biol. Ecol., 350, 46-72.

Cardinale, B.J., Matulich, K.L., Hooper, D.U., Byrnes, J.E., Duffy, E., Gamfeldt, L. et al. (2011). The functional role of producer diversity in ecosystems. Am. J. Bot., 98, 572-592.

Cardinale, B.J., Duffy, J.E., Gonzalez, A., Hooper, D.U., Perrings, C., Venail, P. et al. (2012). Biodiversity loss and its impact on humanity. Nature, 486, 59-67.

Chao, A. \& Jost, L. (2012). Coverage-based rarefaction and extrapolation: standardizing samples by completeness rather than size. Ecology, 93, 2533-2547.

Cloern, J. (2001). Our evolving conceptual model of the coastal eutrophication problem. Mar. Ecol. Prog. Ser., 210, 223-253.

Duffy, J.E., Richardson, J.P. \& Canuel, E.A. (2003). Grazer diversity effects on ecosystem functioning in seagrass beds. Ecol. Lett., 6, 637645 .

Duffy, J.E., Cardinale, B.J., France, K.E., McIntyre, P.B., Thébault, E. \& Loreau, M. (2007). The functional role of biodiversity in ecosystems: incorporating trophic complexity. Ecol. Lett., 10, 522-538.

Duffy, J.E., Moksnes, P.-O. \& Hughes, A.R. (2013). Ecology of Seagrass Communities. In Marine Community Ecology and Conservation. (eds Bertness, M.D., Bruno, J.F., Silliman, B.R., Stachowicz, J.J.). Sinauer Associates, Sunderland, pp. 271-297.

Edgar, G.J. (1990). The use of the size structure of benthic macrofaunal communities to estimate faunal biomass and secondary production. $J$. Exp. Mar. Biol. Ecol., 137, 195-214.

Eriksson, B.K., Ljunggren, L.M., Sandstrom, A., Johansson, G. \& Mattila, J. Rubach, et al. (2009). Declines in predatory fish promote bloom-forming macroalgae. Ecol. Appl., 19, 1975-1988.
Frank, K., Petrie, B. \& Shackell, N. (2007). The ups and downs of trophic control in continental shelf ecosystems. Trends Ecol. Evol., 22, 236-242.

Gamfeldt, L., Snäll, T., Bagchi, R., Jonsson, M., Gustafsson, L., Kjellander, P. et al. (2013). Higher levels of multiple ecosystem services are found in forests with more tree species. Nat. Commun., 4, 1340.

Grace, J.B. (2006). Structural Equation Modeling and Natural Systems. Cambridge University Press, 1-378.

Gruner, D.S., Smith, J.E., Seabloom, E.W., Sandin, S.A., Ngai, J.T., Hillebrand, H. et al. (2008). A cross-system synthesis of consumer and nutrient resource control on producer biomass. Ecol. Lett., 11, $740-755$.

Heck, K.L. \& Valentine, J.F. (2007). The primacy of top-down effects in shallow benthic ecosystems. Estuaries and Coasts, 30, 371-381.

Holt, R.D. \& Loreau, M. (2002). Biodiversity and ecosystem functioning: the role of trophic interactions and the importance of system openness. In The Functional Consequences of Biodiversity. (eds Kinzig, A.P., Pacala, S.W., Tilman, D.). Princeton University Press, Princeton, pp. 246-262.

Hooper, D.U., Adair, E.C., Cardinale, B.J., Byrnes, J.E.K., Hungate, B.A., Matulich, K.L. et al. (2012). A global synthesis reveals biodiversity loss as a major driver of ecosystem change. Nature, 486, $105-108$.

Hughes, A.R. \& Stachowicz, J.J. (2004). Genetic diversity enhances the resistance of a seagrass ecosystem to disturbance. Proc. Natl. Acad. Sci. USA, 101, 8998-9002.

Hughes, A.R., Bando, K.J., Rodriguez, L.F. \& Williams, S.L. (2004). Relative effects of grazers and nutrients on seagrasses: a meta-analysis approach. Mar. Ecol. Prog. Ser., 282, 87-99.

Hughes, B.B., Eby, R., Van Dyke, E., Tinker, M.T., Marks, C.I., Johnson, K.S. et al. (2013). Recovery of a top predator mediates negative eutrophic effects on seagrass. Proc. Natl. Acad. Sci. USA, 110, 15313-15318.

Hunter, M.D. \& Price, P.W. (1992). Playing chutes and ladders: heterogeneity and the relative roles of bottom-up and top-down forces in natural communities. Ecology, 73, 723-732.

Lefcheck, J.S. \& Duffy, J.E. (2015). Multitrophic functional diversity predicts ecosystem functioning in experimental assemblages of estuarine consumers. PeerJ. PrePrints 3:e1137.

Maestre, F.T., Quero, J.L., Gotelli, N.J., Escudero, A., Ochoa, V., Delgado-Baquerizo, M. et al. (2012). Plant Species Richness and Ecosystem Multifunctionality in Global Drylands. Science, 335, 214 218.

Mattson, W.J., Jr. (1980). Herbivory in relation to plant nitrogen content. Annu. Rev. Ecol. Syst., 11, 119-161.

Moksnes, P.O., Gullström, M., Tryman, K. \& Baden, S. (2008). Trophic cascades in a temperate seagrass community. Oikos, 117, 763-777.

Moore, K. \& Short, F. (2006). Zostera: biology, ecology, and management. In: Seagrasses: Biology, Ecology and Conservation (eds. Anthony Larkum, Robert J. Orth, Carlos Duarte), Springer, Dordrecht, pp. 361-386.

Mora, C., Aburto-Oropeza, O., Bocos, A.A., Ayotte, P.M., Banks, S., Bauman, A.G. et al. (2011). Global human footprint on the linkage between biodiversity and ecosystem functioning in reef fishes. PLoS Biol., 9, e1000606.

Nakagawa, S. \& Schielzeth, H. (2013). A general and simple method for obtaining $\mathrm{R}^{2}$ from generalized linear mixed-effects models. Methods Ecol. Evol., 4, 133-142.

O'Connor, M.I. (2009). Warming strengthens an herbivore-plant interaction. Ecology, 90, 388-398.

Oksanen, L. \& Oksanen, T. (2000). The logic and realism of the hypothesis of exploitation ecosystems. Am. Nat., 155, 703-723.

Olsen, J.L., Coyer, J.A., Stam, W.T., Moy, F.E., Christie, H. \& Jørgensen, N.M. (2013). Eelgrass Zostera marina populations in northern Norwegian fjords are genetically isolated and diverse. Mar. Ecol. Prog. Ser., 486, 121-132.

Paquette, A. \& Messier, C. (2010). The effect of biodiversity on tree productivity: from temperate to boreal forests. Global Ecol. Biogeogr., 20, 170-180. 
Polis, G. \& Strong, D. (1996). Food Web Complexity and Community Dynamics. Am. Nat., 147, 813-846.

Poore, A.G.B., Campbell, A.H. \& Steinberg, P.D. (2009). Natural densities of mesograzers fail to limit growth of macroalgae or their epiphytes in a temperate algal bed. J. Ecol., 97, 164-175.

R Core Team. (2014). R: A language and environment for statistical computing. R Foundation for Statistical Computing, Vienna, Austria. Available at http://www.R-project.org/.http://www.R-project.org/.

Reusch, T.B. (2000). Five microsatellite loci in eelgrass Zostera marina and a test of cross-species amplification in $Z$. noltii and $Z$. japonica. Mol. Ecol., 9, 371-373.

Reusch, T.B., Stam, W.T. \& Olsen, J.L. (1999). Microsatellite loci in eelgrass Zostera marina reveal marked polymorphism within and among populations. Mol. Ecol., 8, 317-321.

Reusch, T.B.H., Ehlers, A., Hämmerli, A. \& Worm, B. (2005). Ecosystem recovery after climatic extremes enhanced by genotypic diversity. Proc. Natl. Acad. Sci. USA, 102, 2826-2831.

Reynolds, P.L., Paul Richardson, J. \& Emmett Duffy, J. (2014). Field experimental evidence that grazers mediate transition between microalgal and seagrass dominance. Limnol. Oceanogr., 59, 10531064.

Shipley, B. (2009). Confirmatory path analysis in a generalized multilevel context. Ecology, 90, 363-368.

Shipley, B. (2013). The AIC model selection method applied to path analytic models compared using a d-separation test. Ecology, 94, 560564.

Spivak, A.C., Canuel, E.A., Duffy, J.E. \& Richardson, J.P. (2009). Nutrient enrichment and food web composition affect ecosystem metabolism in an experimental seagrass habitat. PLOS ONE, 4, e7473.

Srivastava, D.S. \& Vellend, M. (2005). Biodiversity-ecosystem function research: is it relevant to conservation? Annu. Rev. Ecol. Evol. Syst., 36, 267-294.
Strong, D.R. (1992). Are trophic cascades all wet? Differentiation and donor-control in speciose ecosystems. Ecology, 73, 747-754.

Tomas, F., Abbott, J.M., Balk, M., Steinberg, C., Williams, S.L. \& Stachowicz, J.J. (2011). Plant genotype and nitrogen loading influence seagrass productivity, biochemistry, and plant-herbivore interactions. Ecology, 92, 1807-1817.

Valentine, J. \& Duffy, J. (2006). The central role of grazing in seagrass ecology. In: Seagrasses: Biology, Ecology, and Conservation (eds. Anthony Larkum, Robert J. Orth, Carlos Duarte), Springer, Dordrecht, pp. 463-501.

Whalen, M.A., Duffy, J.E. \& Grace, J.B. (2013). Temporal shifts in topdown versus bottom-up control of epiphytic algae in a seagrass ecosystem. Ecology, 94, 510-520.

\section{SUPPORTING INFORMATION}

Additional Supporting Information may be downloaded via the online version of this article at Wiley Online Library (www.ecologyletters.com).

Editor, Dr. Boris Worm

Manuscript received 24 November 2014

First decision made 17 December 2014

Second decision made 19 March 2015

Manuscript accepted 2 April 2015 